\title{
Calibration Method for Confocal X-Ray Microanalysis with Polychromatic Excitation
}

\author{
C. Sosa, ${ }^{1}$ V. Stoytschew, ${ }^{2}$ J. Leani, ${ }^{1}$ H. J. Sánchez, ${ }^{1,3}$ C. A. Pérez, ${ }^{4}$ and R. D. Perez ${ }^{1,3}$ \\ ${ }^{1}$ CONICET, Rivadavia 1917, 1033 Buenos Aires, Argentina \\ ${ }^{2}$ Institute for Optics and Atomic Physics, Technische Universität Berlin, Hardenbergstrasse 36, 10623 Berlin, Germany \\ ${ }^{3}$ FaMAF, Universidad Nacional de Córdoba, Ciudad Universitaria, 5000 Córdoba, Argentina \\ ${ }^{4}$ Laboratorio Nacional de Luz Síncrotron (LNLS), P.O. Box 6192, 13084-971 Campinas, SP, Brazil
}

Correspondence should be addressed to R. D. Perez; danperez@famaf.unc.edu.ar

Received 8 October 2014; Revised 19 December 2014; Accepted 23 December 2014

Academic Editor: Rafal Sitko

Copyright $(2015$ C. Sosa et al. This is an open access article distributed under the Creative Commons Attribution License, which permits unrestricted use, distribution, and reproduction in any medium, provided the original work is properly cited.

\begin{abstract}
To apply the fundamental parameters method at the confocal setup the knowledge of the sensitivity of the spectrometer is required which depends on the characteristics of two X-ray lenses: one in the excitation channel and another in the detection channel. For the particular case of polychromatic excitation, the theory shows that the focalization properties of the excitation lens for all incident energies affect the X-ray fluorescence intensity. Therefore the traditional calibration method based on the measurement of standard samples becomes unstable since the number of required fitting parameters is too high. To reduce these parameters a previous characterization of the excitation lens by a simulation program was employed giving rise to a simplified confocal setup calibration. The developed calibration method was applied for a confocal spectrometer implemented in the Brazilian Synchrotron Radiation Source (LNLS) with white beam. The experimental parameters of the sensitivity were obtained from depth profile analysis of several pure thin films. The calibrated confocal setup was used to quantify reference standards in order to validate the calibration procedure. Our results for elemental concentrations show relative errors less than $15 \%$ for the quantitative analysis of a light matrix reference standard.
\end{abstract}

\section{Introduction}

A new branch in the X-ray spectrometry is the 3D microanalysis in confocal configuration (3D-MXRF). It uses the same principles as conventional X-ray fluorescence (XRF) technique but it adds $\mathrm{X}$-ray lenses in the excitation and detection channels. The characteristic radiation coming from a microvolume defined by the overlap of the foci of both Xray lenses is ideally analyzed. The fundamental parameter (FP) method applied for the mathematical description of 3D-MXRF is based on the three-dimensional model for the probing volume [1]. The model describes the sensitivity of the spectrometer which relates the theoretical and measured XRF intensities in every pixel of the sample. For the particular case of polychromatic excitation, an additional energy integral is required to evaluate the XRF intensity. Consequently, elemental quantification becomes a difficult task which can be avoided by the monochromatization of the X-ray source.
However, the inclusion of a monochromator considerably reduces the intensity of polychromatic X-ray sources while it increases the acquisition time in spatial mappings. It is a serious drawback for 3D-MXRF technique especially when X-ray tube excitation is employed. Then, to efficiently extend the application of the 3D-MXRF to conventional X-ray sources it is desirable to research the possible analytical approaches in spite of the inherent difficulties in data analysis.

The first step for the application of FP to the 3DMXRF technique is the calibration of the spectrometer which consists of the spectrometer sensitivity determination. For polychromatic excitation the model for the sensitivity shows that the influence of the excitation lens in the XRF intensity is more pronounced, since their focalization properties for all energies present in the incident beam are involved in the theoretical description. When the traditional calibration method for FP based on measurement of standard samples is applied, the fitting of the theoretical model leads to 
indeterminate solutions for the sensitivity. Furthermore, if the initial values for the fitting parameters are not close to the solution then the fitting process becomes unstable. To overcome this problem a previous characterization of the excitation lens is recommended. It can be directly done by scanning the energy of a tunable monochromatic beam through the excitation lens and measuring the spatial distribution of photon beam in its focus. The implementation of this procedure could be difficult in a conventional laboratory where usually the X-ray source hardly admits the inclusion of a monochromator. Another possibility is the application of a theoretical model to calculate the radiation transport through the lens. Since the proven model for this calculation is based on multiple total reflections of X-ray photons in the internal walls of the lens, it is difficult to deduce what kind of energy functions describes the behavior of capillary lenses. Effectively, the calculation requires following all the interaction events of X-rays through the lens [2]. For this reason practical application of the theoretical model requires computer simulation.

In our calibration process we scan pure thin films through the probing volume of the confocal spectrometer as is proposed in a previous publication [3]. Then we calculate the spectrometer sensitivity for depth profile analysis by fitting the theoretical XRF intensities to the measurements. However, we considered the focalization properties of the excitation lens as a known data in order to reduce the fitting parameters. We obtained these data from simulations carried out by our own programming code written in FORTRAN language. The proposed calibration method avoids divergences in the calculation giving rise to a robust fitting process. The accuracy of the methodology was tested using the calculated sensitivity for the elemental quantification of a light matrix reference standard.

\section{Materials and Methods}

2.1. Instrumentation. The experiment was carried out in the D09B beamline of the Synchrotron Light National Laboratory (Laboratório Nacional de Luz Síncrotron, LNLS) using white beam [4]. In our calculations we employed the synchrotron spectrum measured by technical operators of the beamline. A silicon drift X-ray detector with $150 \mathrm{eV}$ of resolution at $5.9 \mathrm{keV}$ was positioned perpendicular to the photon beam on the horizontal plane. This system was mounted on a motorized XYZ stage. Suspended from the snout of the silicon drift detector, a fixed holder holds a commercial half monolithic polycapillary with its optical axes centered and normal to the window of the detector. This polycapillary has a focal distance of $16 \mathrm{~mm}$ with a beam size of $20 \mu \mathrm{m}$ FWHM and a gain of 600 at the mean energy of the white beam. In the excitation channel a conical glass monocapillary was mounted in a special motorized gimbal. The distance from the output of the monocapillary to the focal spot of the half monolithic polycapillary was $15 \mathrm{~mm}$. The mean transmission efficiency of the monocapillary for white beam is $34 \%$. Transmission dependence with the incident energy was determined by means of a simulation program as is

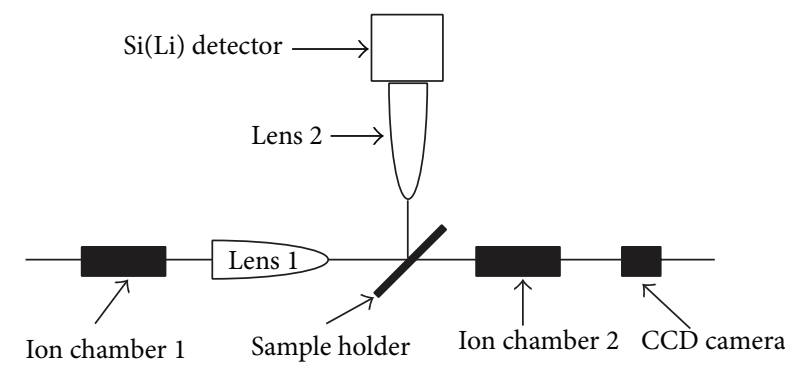

FIGURE 1: Experimental setup of the confocal spectrometer in the LNLS.

further described. Samples were mounted vertically at $45^{\circ}$ of the incident direction on a motorized XYZ sample stage with a spatial resolution of $0.6 \mu \mathrm{m}$. A diagram of the experimental array is shown in Figure 1.

Ionization chambers at the entrance of the monocapillary and behind the sample holder were used to align efficiently the monocapillary. In addition, a CCD camera was placed behind the sample holder to determine the position of the incident beam. A digital optical microscope focused on the sample was employed to distinguish details on the area excited by the incident beam.

2.2. Measurements and Calibration of $3 D-M X R F$. To calibrate the 3D-MXRF setup for depth profile analysis, we perform linear scans through the normal direction of pure thin films. We used thin film standards (Micromatter Co.) of $\mathrm{ClK}, \mathrm{Ti}, \mathrm{Mn}, \mathrm{Fe}, \mathrm{Cu}$, and $\mathrm{Zn}$ with high purity and known thickness. For all scans, the counting live-time for each point was $10 \mathrm{~s} / \mathrm{step}$ and the step size was $10 \mu \mathrm{m}$. For all elements we followed the K $\alpha$ X-ray fluorescent line. To evaluate the resulting spectra, the AXIL package [5] was employed. Mass attenuation coefficients were taken from McMaster et al. [6].

Once the calibration was established, we performed a quantitative analysis by $3 \mathrm{D}-\mathrm{MXRF}$ of $\mathrm{K}, \mathrm{Mn}, \mathrm{Fe}, \mathrm{Cu}$, and $\mathrm{Zn}$ for bovine liver standard material $1577 \mathrm{~b}$ provided by the National Institute of Standards \& Technology (NIST). It was prepared as pressed pellet without any additional binder. Linear scans through the normal direction on three different locations of the sample were performed in order to study its homogeneity.

2.3. Analytical Simulation of Excitation Lens. Radiation transport simulations in the excitation lens were performed using our own program code that allows loading the synchrotron beam spectrum as well as the geometry of the capillary and the experimental conditions. It was written in FORTRAN language considering the multiple reflections of the incident beam in the walls of the excitation lens and the probability of external total reflection. The main advantage of this program is that it takes into account the transmission of the radiation through the capillary wall. Therefore, this contribution is considered when calculating the profile of the radiation beam at the exit of the capillary. The simulations were employed to obtain the energy dependence of the transmission and focus sizes of our excitation lens. 


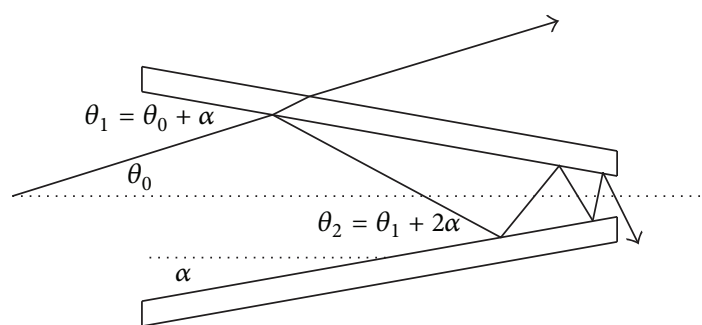

FIGURE 2: Schematic diagram of the radiation transport through the conical monocapillary.

For the sake of simplicity the X-ray source was considered in good approximation as a point source, since the entrance slits of the beamline are placed $15 \mathrm{~m}$ far from the capillary giving rise to an almost parallel beam with $0.2 \mathrm{mrad}$ of divergence. When the capillary is aligned the point source is on the symmetry axis, which means that the radiation transport simulation can be reduced in two dimensions maintaining the precision of the calculations. The capillary was modelled by a conical profile of $3 \mathrm{mrad}$ inclination made of borosilicate glass according to the manufacturer's specifications (see Figure 2). Its dimensions were $235 \mathrm{~mm}$ length, $0.075 \mathrm{~mm}$ input inner diameter, $5 \mathrm{~mm}$ input outside diameter, $0.013 \mathrm{~mm}$ output inner diameter, and $0.85 \mathrm{~mm}$ output outside diameter.

The simulation begins with the input of photons into the capillary considering all the possible directions and energies from the source in one plane generated by the symmetry axis of the lens. Depending on the incident angle they can be reflected or refracted in the glass interface. The low inclination angle of the conical capillary assures a high probability of total external reflection which is responsible for multiple reflections into the lens. The reflection and refraction beams in the glass interface have been described by means of the approximate model introduced by Parrat [7]. Within this approximation, no distinction needs to be made between polarization states of the incident beam which is confirmed by a direct analysis of the beam propagation at grazing incidence [8]. Then, when a photon of energy $E$ impinges in a point $p$ on the glass wall of the capillary, the probability to be reflected can be written as

$$
R(\theta, E)=\left|\frac{\theta-\sqrt{\theta^{2}-\theta_{c}^{2}-2 i \beta}}{\theta+\sqrt{\theta^{2}-\theta_{c}^{2}-2 i \beta}}\right|^{2},
$$

where $\theta$ is the angle between the incident direction and the inner wall of the capillary, $\theta_{c}$ is the total external reflection angle, $i$ is the imaginary number, and $\beta$ takes into account the attenuation in the glass by means of

$$
\beta=\frac{h c}{4 \pi} \frac{\mu(E) \rho}{E}
$$

where $h$ is Planck's constant, $c$ the light velocity, $\rho$ the mass density of glass, and $\mu(E)$ the mass attenuation coefficient of glass at energy $E$.
According to the reflection law the incident angle for the next reflection is $\theta^{\prime}=\theta+2 \alpha$, where $\alpha$ is the inclination angle of the conical profile. However, the photon at point $p$ could not be reflected but it is refracted in which case the transmission probability through the interface is given by

$$
T(\theta, E)=\left|\frac{2 \theta}{\theta+\sqrt{\theta^{2}-\theta_{c}^{2}-2 i \beta}}\right|^{2} .
$$

If the photon arrives at point $p$ by $\delta$ previous reflections then the total probability to be reflected or transmitted is given by

$$
P(E)=\prod_{j=1}^{\delta} R\left(\theta_{j}, E\right) e^{-\mu_{\mathrm{air}} t_{j}} \times X(\theta, E),
$$

where $X(\theta, E)=R(\theta, E)$ for reflection and $X(\theta, E)=T(\theta, E)$ for transmission. The incident angle at the $j$ reflection is $\theta_{j}$. In the equation the air attenuation in the radiation path $t_{j}$ between the $j$ and $(j+1)$ reflections was included.

The above are the main equations employed in the programming code for the radiation transport simulation in the excitation lens. As was mentioned before, it was employed to evaluate the focalization characteristics of the lens described by its size and transmission. The transmission was calculated as the ratio of the number of photons leaving the lens to the number of photons reaching its entrance. Considering monochromatic beams with different energies, it was possible to obtain the energy dependence of the size and transmission of the lens.

\section{Theory}

Assuming a homogeneous film of thickness $D$, the intensity of a specific X-ray line of an element $i$ excited by a polychromatic source as a function of the normal coordinate $x$ can be written extending the model described in [1] as

$$
\begin{aligned}
I p_{i}(x)=\int_{0}^{E_{m}} & \rho_{i} I_{0}(E) \tau_{F, i}(E) \\
& \times\left(\int_{0}^{D} \eta_{i}\left(E, x^{\prime}-x\right) \exp \left(-\widetilde{\mu}_{i}(E) x^{\prime}\right) d x^{\prime}\right) d E,
\end{aligned}
$$

where $E_{m}$ is the maximum energy of the incoming photons, $I_{0}(E)$ is the incoming photon flux of energy $E, \rho_{i}$ is the density (in $\mathrm{g} / \mathrm{cm}^{3}$ ) of the $i$-element in the sample, $\tau_{F, i}$ is the production cross section (in $\mathrm{cm}^{2} / \mathrm{g}$ ) for the measured X-ray line of the $i$-element at energy $E, \eta_{i}(E, x)$ is the sensitivity profile of the spectrometer for the $i$-element, and $\widetilde{\mu}_{i}$ is the effective linear mass attenuation coefficient at energy $E$ for the $i$ element defined as follows:

$$
\widetilde{\mu}_{i}=\sum_{j=1}^{r} \rho_{j}\left(\frac{\mu_{j}(E)}{\sin \left(\theta_{0}\right)}+\frac{\mu_{j}\left(E_{i}\right)}{\sin \left(\theta_{1}\right)}\right),
$$


where $\rho_{j}$ is the density of the $j$-element, $\mu_{j}(E)$ is the mass attenuation coefficient of the $j$-element at energy $E, E_{i}$ is the energy of the X-ray line of the $i$-element, and $\theta_{0}$ and $\theta_{1}$ are the medium angle of the impinging beam and detected beam, respectively.

The sensitivity profile can be written as

$$
\eta_{i}(E, x)=k_{i} f_{i}(E) \exp \left(\frac{-x^{2}}{2 \sigma_{i}(E)^{2}}\right)
$$

where

$$
\begin{gathered}
k_{i}=\frac{\Omega \varepsilon\left(E_{i}\right) \sigma_{D}^{2}\left(E_{i}\right) T_{D}\left(E_{i}\right)}{2 \pi}, \quad f_{i}(E)=\frac{T_{A}(E)}{\sigma_{i}^{2}(E)}, \\
\sigma_{i}(E)=\sqrt{\left(\frac{\sigma_{A}(E)}{2}\right)^{2}+\left(\frac{\sigma_{D}\left(E_{i}\right)}{2}\right)^{2}}
\end{gathered}
$$

$\Omega$ is the solid angle accepted by the lens in the detection channel, $\varepsilon$ is the detector efficiency, $T_{A}$ and $T_{D}$ are the transmission efficiencies of the lenses in the excitation and detection channel, respectively, and $\sigma_{A}$ and $\sigma_{D}$ represent the focal sizes of the lenses in the excitation and detection channel, respectively.

In particular, for a very thin film of thickness $d$ we have

$$
I p_{i}(x)=\int_{0}^{E_{m}} \rho_{i} I_{0}(E) \tau_{F, i}(E) \eta_{i}(E, x) d .
$$

Thus, through a vertical scan of a reference standard thin film of knowledge thickness and composition, it is possible in principle to determine the sensitivity of the spectrometer. However, the dependence of $\sigma_{A}$ and $T_{A}$ with the excitation energy could add enough fitting parameters for the calculation to become unstable. The basic idea for overcoming the instability is the employment of the results of a previous characterisation of the excitation lens. Then, the fitting parameters are reduced to $k_{i}$ and $\sigma_{D}\left(E_{i}\right)$ which can be unambiguously determined.

\section{Results and Discussions}

The simulation of the radiation transport shows that all photons incident at the entrance of capillary are in the total reflection regime. It occurs even for $20 \mathrm{keV}$ photons which are the higher energy useful for XRF excitation in the beamline. It means that the lens has a high transmission for the energy range of employed synchrotron source. Furthermore, the analysis of the photon trajectories shows that $17 \%$ of them pass through the capillary without interactions, and 34\% suffered one reflection, another $34 \%$ two consecutive reflections, and the remaining $15 \%$ three consecutive reflections. Reflections of higher orders were not observed even for the largest incident angles. In this lens the transmitted photons through the glass wall of the capillary are minimum since the interactions are always under the total reflection regime. As an example of the efficiency of the lens, Figure 3 shows the probability of total reflection for photons at third reflection with largest incident angles at the total reflection regime. It

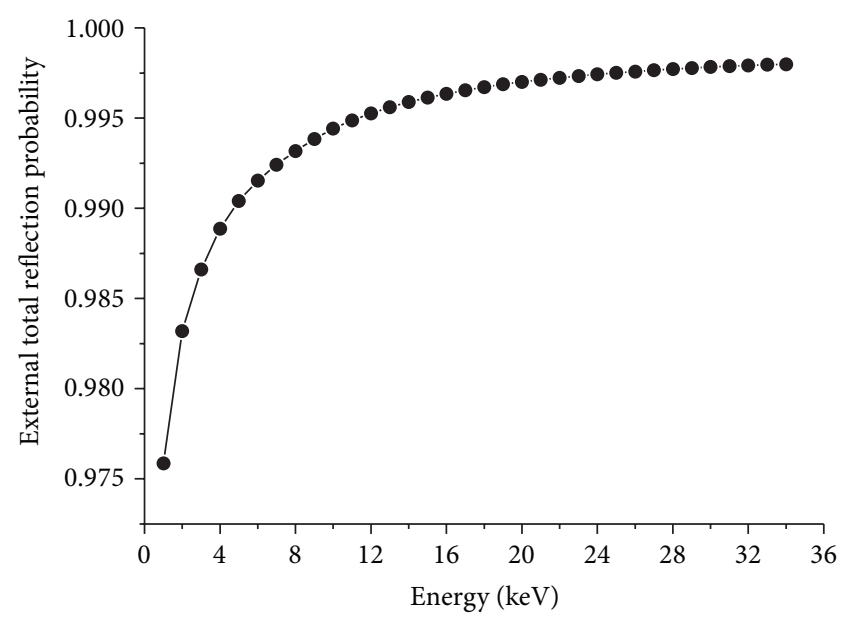

Figure 3: Probability of total reflection as function of energy, for photons at the third reflection with the largest incident angle in the total reflection regime. It was calculated through (4) taking $\delta=2$ and the incident angles at each reflection equal to the total reflection angle.
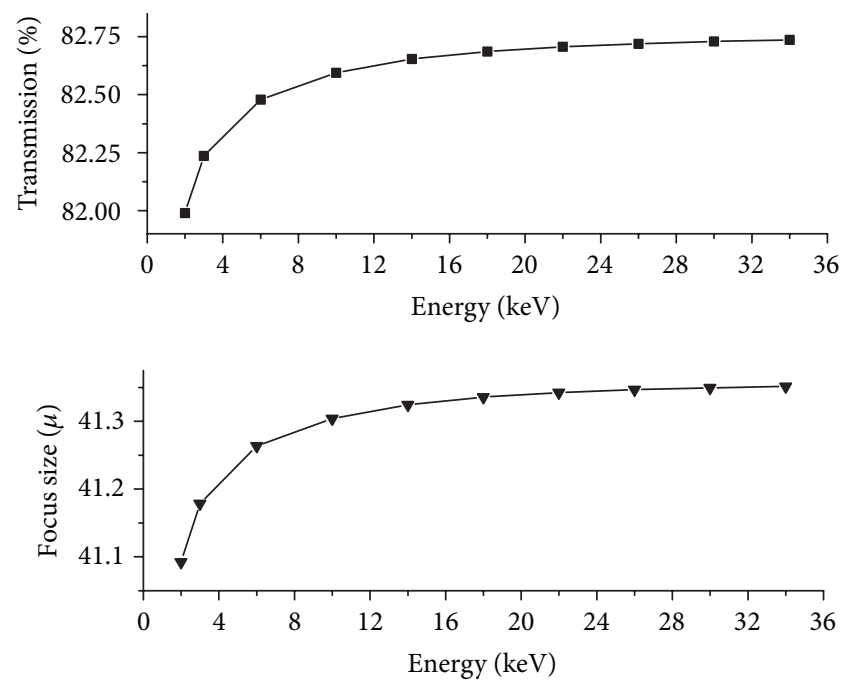

FIgURE 4: Percent transmission and focus size of the excitation channel lens at $15 \mathrm{~mm}$ from its output versus the incident energy. Here the focus size is represented by the FWHM of the transversal cross section of the emergent beam. It is given by product of $\sigma_{A}$ by 2.35482 .

was calculated through (4) taking $\delta=2$ and the incident angles at each reflection equal to the total reflection angle. The results are close to the ideal curve through all energy range with a slight decrease for lower energies caused by $\mathrm{X}$ ray attenuation in the glass wall.

Figure 4 shows the transmission and focus size of the lens for various energies at excitation distance. It can be seen that the difference of the focus size and transmission between 1 and $35 \mathrm{KeV}$ is less than $1 \%$. Since the practical application range for the synchrotron radiation emission of the LNLS is approximately until $20 \mathrm{keV}$, we can assume that the characteristics of the excitation lens employed in 


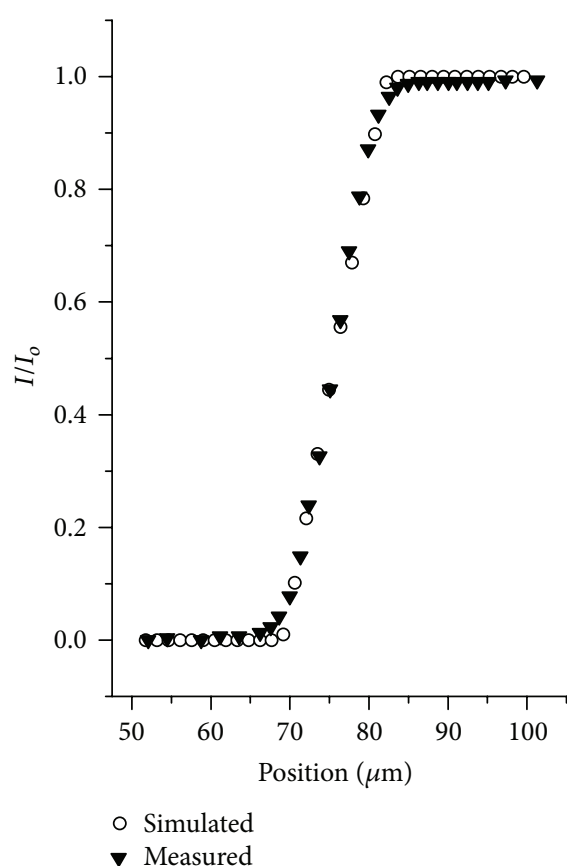

(a)

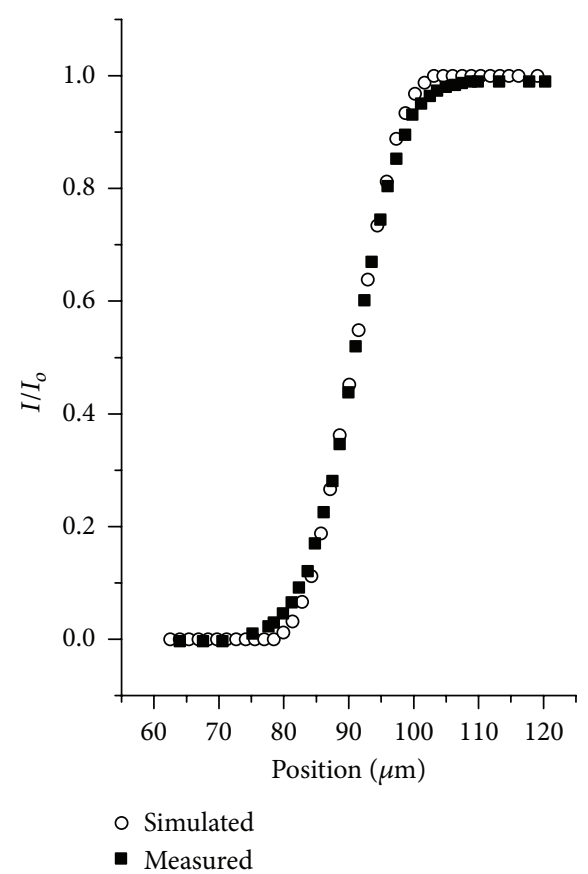

(b)

FIGURE 5: Measured and simulated vertical profile of the microbeam: (a) at $1 \mathrm{~mm}$ and (b) at $10 \mathrm{~mm}$. In (a) the mean FWHM for the simulated beam is $22.1 \mu \mathrm{m}$ and the measurement gives $22.3 \mu \mathrm{m}$. For (b) the mean FWHM for the simulated beam is $34.5 \mu \mathrm{m}$ and the measurement gives $34.7 \mu \mathrm{m}$.

TABLE 1: Sensitivity parameters and validation of the calibration.

\begin{tabular}{|c|c|c|c|c|c|c|c|}
\hline & \multicolumn{2}{|c|}{ Sensitivity parameters } & \multicolumn{5}{|c|}{$\begin{array}{c}\text { Quantification of NIST1577b } \\
\text { Bovine liver }\end{array}$} \\
\hline & $\begin{array}{c}k_{i} * f_{i} \\
{\left[\times 10^{-36}\right]}\end{array}$ & $\begin{array}{c}\sigma_{i} \\
{[\mu \mathrm{m}]}\end{array}$ & $\begin{array}{l}\text { Certified } \\
\text { [ppm] }\end{array}$ & $\begin{array}{l}\text { Point } 1 \\
{[\mathrm{ppm}]}\end{array}$ & $\begin{array}{r}\text { Point } 2 \\
\text { [ppm] }\end{array}$ & $\begin{array}{c}\text { Point } 3 \\
\text { [ppm] }\end{array}$ & $\begin{array}{l}\text { Average } \\
{[\mathrm{ppm}]}\end{array}$ \\
\hline $\mathrm{K}$ & 2.46 & 39 & 9940 & 9100 & 8900 & 8700 & $9100 \pm 200$ \\
\hline Mn & 13.6 & 34 & 10.5 & - & - & - & - \\
\hline $\mathrm{Fe}$ & 14.8 & 33 & 184 & 133 & 165 & 170 & $156 \pm 20$ \\
\hline $\mathrm{Cu}$ & 15.2 & 29 & 160 & 125 & 140 & 156 & $140 \pm 16$ \\
\hline $\mathrm{Zn}$ & 16.3 & 27 & 127 & 97 & 110 & 123 & $110 \pm 13$ \\
\hline
\end{tabular}

The first two columns show the sensitivity parameters for the calibrated elements. The last four columns show the quantification of the reference standard NIST1577b by the confocal setup applying the proposed calibration approach on three different points of the prepared standard.

our setup are independent of the incident energy which simplifies the fitting of the spectrometer sensitivity. However, it is important to highlight that even in our advantageous setup with sensitivity independent of the incident energy, the previous characterization of the excitation lens is a strongly required one. Effectively, the simulation justifies our simplification in the theoretical model of the sensitivity.

To validate the developed program code, measurements of the microbeam cross section at different distances from the output of the capillary were compared with simulations. The profile of the microbeam was obtained by scanning a gold knife-edge throughout its cross section. The incident and transmitted intensity were simultaneously measured using ionization chambers before and after the knife-edge. The same experimental conditions were simulated with our program in FORTRAN. Figure 5 shows the comparative results for vertical scans at 1 and $10 \mathrm{~mm}$ from the output of the lens.

Figure 6 shows the linear scan through the normal direction of thin film and bulk reference standard NIST $1577 \mathrm{~b}$ and the fitting of the theoretical model. Similar linear scans on thin films and reference standard were fitted for all elements calibrated in the spectrometer. The first two columns of Table 1 show the sensitivity parameters of the analyzed elements for $\mathrm{K} \alpha$ lines. In spite of the fact that the focalization properties of the excitation lens are constant with energy, a variation of the sensitivity parameters is observed. Its theoretical equations show that the detection lens produces the observed changes. As was previously studied for polycapillary lenses in collecting mode [9], the focus size gradually 


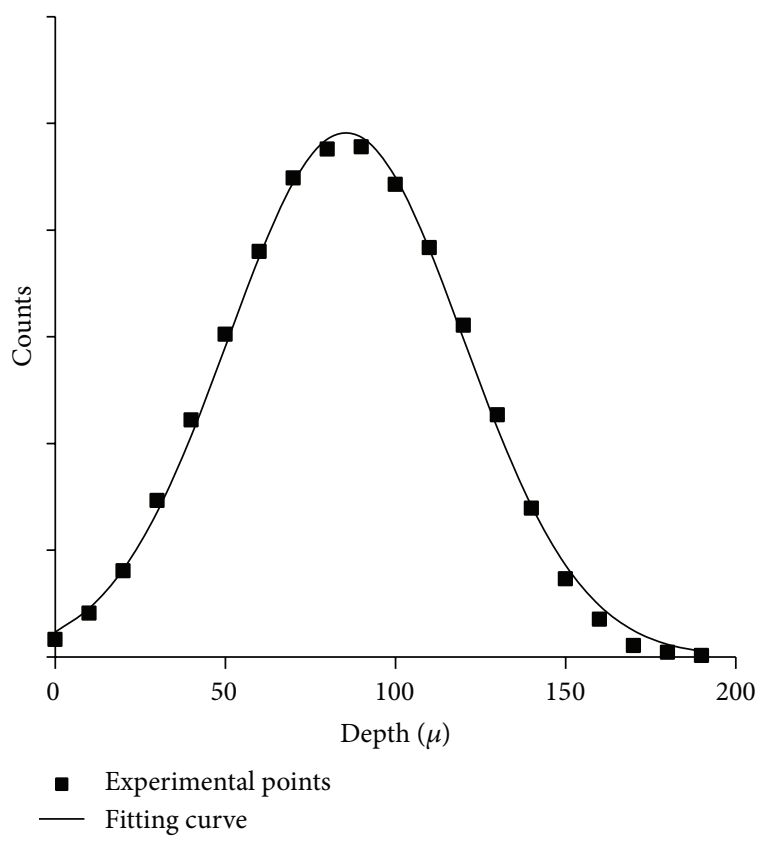

(a)

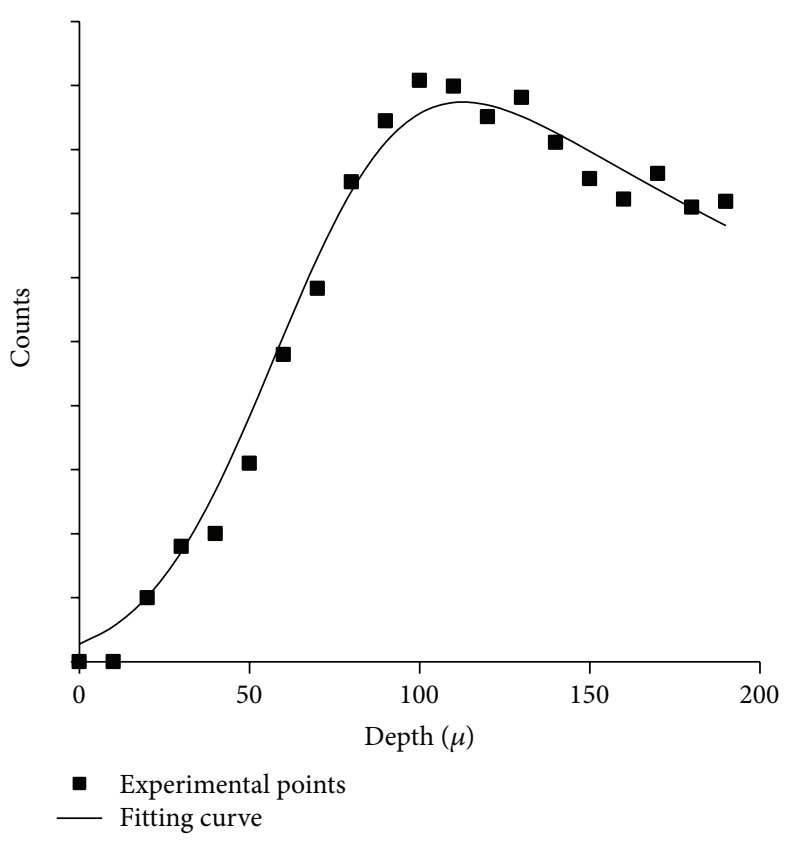

(b)

FIGURE 6: (a) Linear scan through the normal direction of Ti thin film by our confocal spectrometer. The continuous curve represents the fitting of the theoretical model. (b) Similar linear scan for Fe in the standard reference sample NIST 1577b.

decreases with energy and the transmission has a strong increase with a maximum value for approximately $7 \mathrm{keV}$. A similar dependence was observed for the confocal focus size and the proportional factor of the confocal sensitivity which justifies the theoretical model applied in our work.

The last four columns of Table 1 show the quantification results for the light matrix reference standard using the sensitivity determined by thin film scans. A quantification procedure based on an iterative algorithm was employed as in a previous publication [3]. Since for light matrices the detection of X-ray fluorescence emission of light elements is technically not feasible, the algorithm is not self-sufficient and requires an estimation of the density of each undetected light element present in the sample. For the bovine liver reference material the manufacturer does not include information of the matrix composition. Considering that liver is composed mainly of proteins we adopted typical values for the mass fraction of its major elements: carbon $53 \%$, hydrogen $6 \%$, oxygen $23 \%$, and nitrogen $18 \%$ [10]. The volumetric density of the prepared standard sample was obtained by a direct measurement of the mass and volume of the pressed pellet.

As confocal micro-XRF gives elemental volumetric concentrations, they were divided by the volumetric density previously determined to compare with the certified mass concentrations. The results obtained for three different locations in the sample are shown in Table 1. The relative standard deviations for the mass concentrations were less than $13 \%$ showing that the homogeneity of the sample is maintained at the confocal microvolume dimensions. It is expected since the particle size distribution for the bovine liver standard determined by the manufacturer has $50 \%$ of the size of particles less than 7.57 microns and 90\% less than 28.5 microns.

The mean values for the mass concentrations are slightly smaller than the nominal concentrations but the relative discrepancies are less than $15 \%$. We attribute these differences to misalignments of the standard samples in the sample holder since we used a specific support for them which was different to the thin film standard support. At present we are improving the sample holder to correct this effect.

Finally, the quantification of a paint layer on a glass substrate was done applying the present calibration process. The results were published in a previous paper as validation of a proposed elemental quantification approach for intermediate thick layers [3]. For the near future we are going to compare our procedures with new approaches recently presented in the scientific literature $[11,12]$.

\section{Conclusions}

The calibration of the confocal setup with polychromatic excitation requires a careful characterization of the focalization properties of the excitation lens. The characterization of the lens in the detection channel can be overcome since their parameters are unambiguously fixed in the calibration. Our results show that the calibration process based on the minimization of fitting parameters by means of simulations of excitation lens is recommended for polychromatic X-ray sources to avoid the beam monochromatization required by a typical experimental characterization. It is an efficient lowcost solution which can be implemented in conventional Xray laboratories. Furthermore, the accuracy of the standard 
quantifications obtained in our work confirms the fundamental parameter model applied to the confocal geometry.

\section{Conflict of Interests}

The authors declare that there is no conflict of interests regarding the publication of this paper.

\section{Acknowledgments}

Part of the work was carried within the framework of a Coordinated Research Project of the International Atomic Energy Agency (RC-16762). Other institutions involved are CONICET and MINCyT. The authors are grateful to W. Malzer, I. Mantouvalou, and B. Kangiesser from the Institute for Optics and Atomic Physics, Technische Universität Berlin, Germany, for careful reading of the paper and valuable comments.

\section{References}

[1] W. Malzer and B. Kanngieer, "A model for the confocal volume of 3D micro X-ray fluorescence spectrometer," Spectrochimica Acta Part B: Atomic Spectroscopy, vol. 60, no. 9-10, pp. 1334-1341, 2005.

[2] H. J. Sánchez and C. A. Pérez, "Calculation of capillary parameters for the characterization of X-raymicrobeams," Journal of Trace and Microprobe Techniques, vol. 16, pp. 501-512, 1998.

[3] R. D. Perez, H. J. Sánchez, M. Rubio, and C. A. Perez, "Analysis of thin intermediate layers by confocal $\mu$-XRF," X-Ray Spectrometry, vol. 40, no. 1, pp. 19-23, 2011.

[4] C. A. Pérez, M. Radtke, H. J. Sánchez et al., "Synchrotron radiation $x$-ray fluorescence at the LNLS: beamline instrumentation and experiments," X-Ray Spectrometry, vol. 28, no. 5, pp. 320326, 1999.

[5] R. E. van Grieken and A. A. Markowicz, "Chapter 6. Quantification in XRF analysis of intermediate-thickness samples," in Handbook of X-Ray Spectrometry, R. E. van Grieken and A. A. Markowicz, Eds., Marcel Dekker, New York, NY, USA, 2nd edition, 2002.

[6] W. H. McMaster, N. Kerr del Grande, J. H. Mallet, and J. Hubbell, "Compilation of X-ray cross section," Tech. Rep. UCRL 50174, Sect 2, Rev 1, Lawrence Livermore National Laboratory, Livermore, Calif, USA, 1969.

[7] L. G. Parrat, "Surface studies of solids by total reflection of Xrays," Physical Review, vol. 95, pp. 359-369, 1954.

[8] M. Born and E. Wolf, Principles of Optics, chapter 1, Pergamon Press, New York, NY, USA, 6th edition, 1980.

[9] T. Wolff, I. Mantouvalou, W. Malzer et al., "Performance of a polycapillary halflens as focussing and collecting optic-a comparison," Journal of Analytical Atomic Spectrometry, vol. 24, no. 5, pp. 669-675, 2009.

[10] U. Satyanarayana and U. Chakrapani, Biochemistry, chapter 4, Elsevier, New Delhi, India, 4th edition, 2013.

[11] T. Schoonjans, G. Silversmit, B. Vekemans et al., "Fundamental parameter based quantification algorithm for confocal nano-Xray fluorescence analysis," Spectrochimica Acta Part B: Atomic Spectroscopy, vol. 67, pp. 32-42, 2012.

[12] P. Wrobel and M. Czyzycki, "Direct deconvolution approach for depth profiling of element concentrations in multi-layered materials by confocal micro-beam X-ray fluorescence spectrometry," Talanta, vol. 113, pp. 62-67, 2013. 

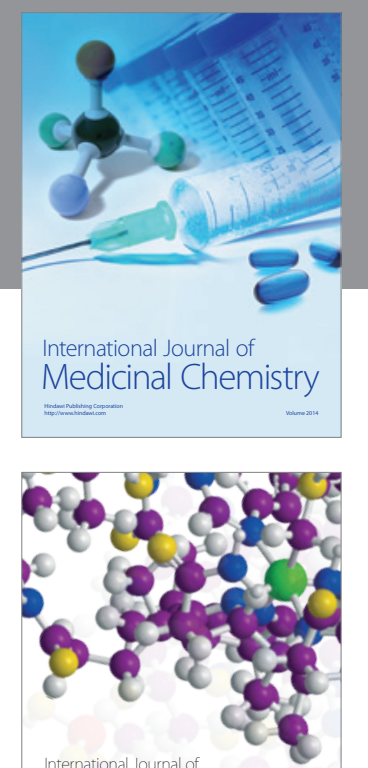

\section{Carbohydrate} Chemistry

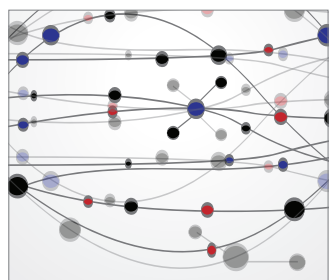

The Scientific World Journal
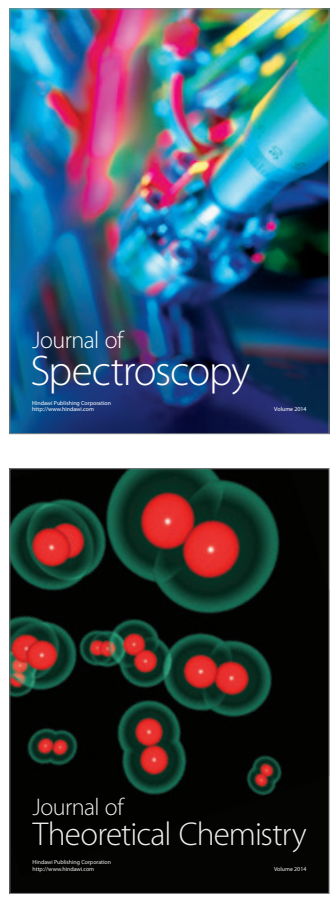
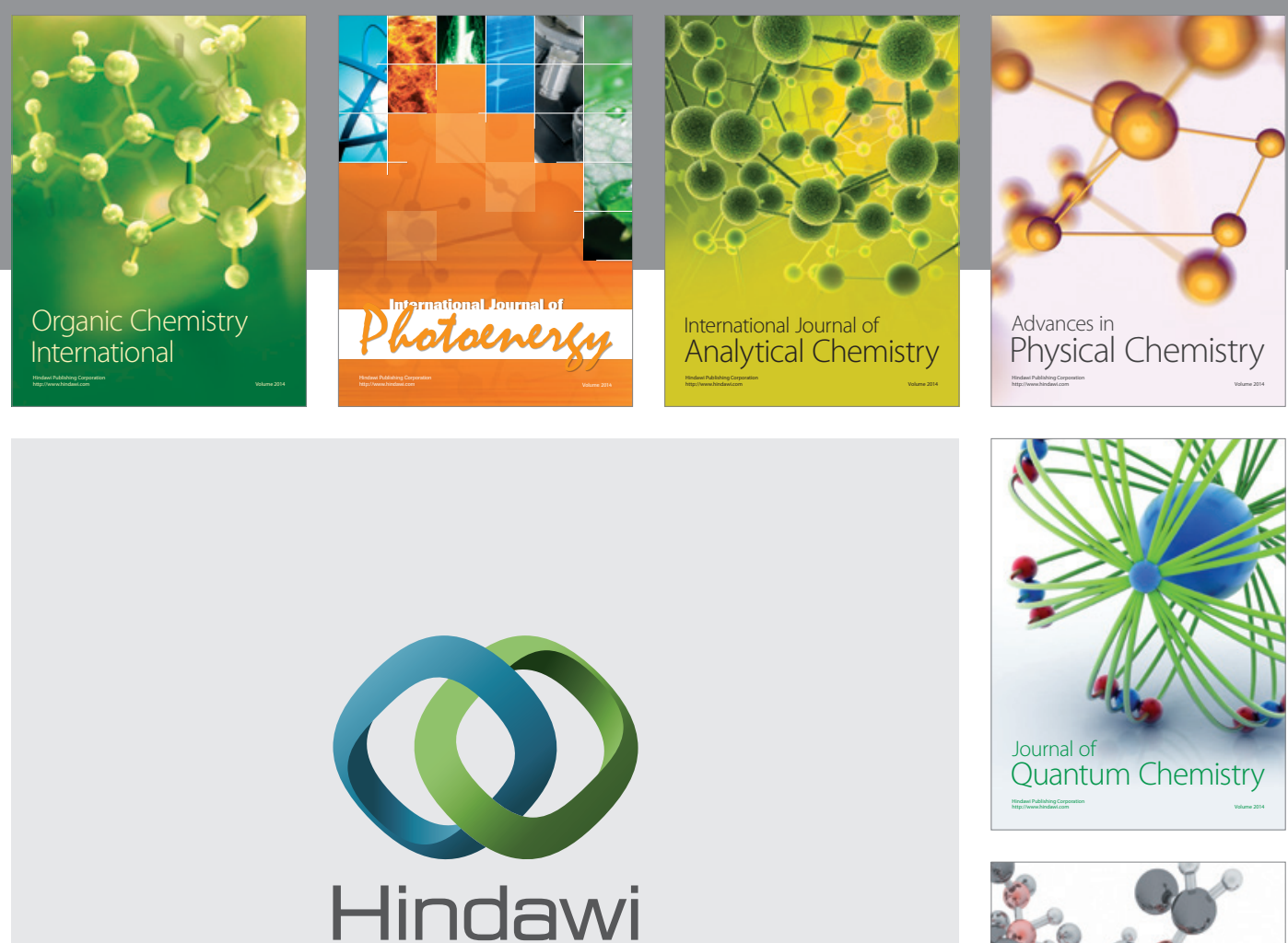

Submit your manuscripts at

http://www.hindawi.com

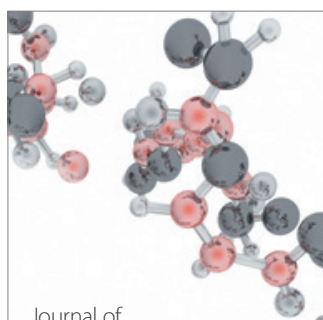

Analytical Methods

in Chemistry

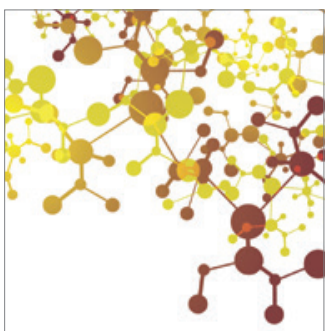

Journal of

Applied Chemistry

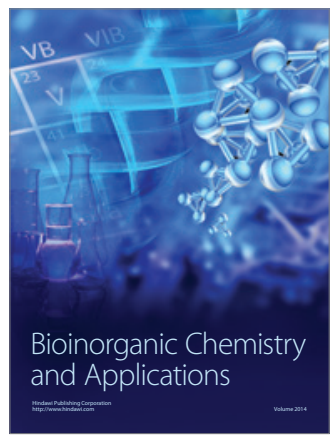

Inorganic Chemistry
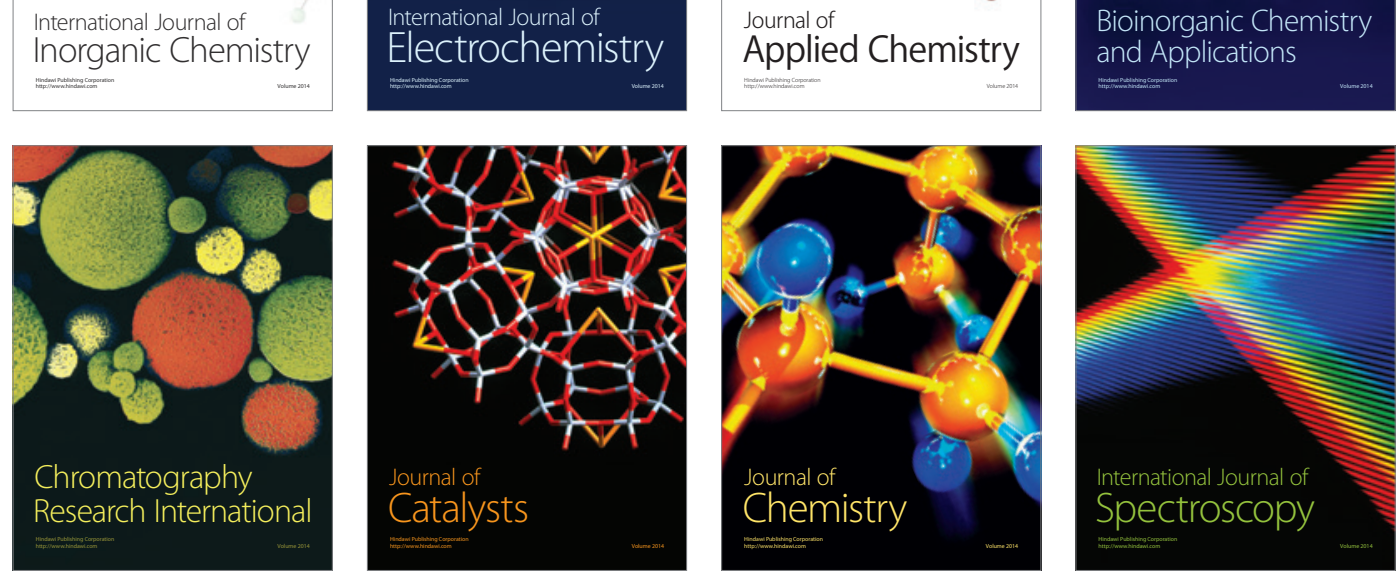